\title{
Legionella pneumophila: risk assessment and strategy for the prevention and control of nosocomial infections
}

\author{
Salvo Torrisi', Eletta Cavedoni', Ornella Pastoris², Chiara Brema', Manuela Verri², Andria Innocenza \\ Bongiorno², Maurizia Dossena², Michela Benazzo', Eleonora Sala', Luciano Cavedoni' \\ I Cosmolab srl \\ 2 Dipartimento di Medicina Legale, Scienze Forensi e Farmaco-Tossicologiche “A. Fornari”, Sez. Scienze Farmacologiche e \\ Tossicologiche, Università degli Studi di Pavia
}

Key words: Legionella pneumophila, Legionellosi, Rischio, Prevenzione

Legionella pneumophila: a valutazione del rischio e strategie per la prevenzione e il controllo delle infezioni nosocomiali

\section{SUMMARY}

The term "Legionellosis" includes all forms of disease caused by microorganisms of the genus Legionella; it may manifest as a flu-like shape (Pontiac fever), or with severe pneumonia with high mortality (Legionnaires Disease). The causative agent was Legionella pneumophila in the literature although other strains of the genus Legionella are classified as pathogens, mode of transmission is through inhalation of aerosol particles produced by hot water or air conditioning systems: for this reason in community settings and nosocomial L. pneumophila represents a serious public health problem. In the light of epidemiological data since the year 2000 the Italian State has issued a series of provisions laws concerning the prevention and control of nosocomial Legionellosis environment and community. The present work aims to evaluate the presence of Legionella species and L. pneumophila comparing the different approaches proposed by the Guidelines of the regions of Lombardy and Piedmont in terms of assessment and prevention of risk "Legionellosis" in the field of nosocomial infection. The analytical methods used are those provided by the Regional Guidelines: the official method in the second CSR April 4 Method 2000 and UNI EN ISO II73I-I: 2008.

Checks have been performed on equipment for the comparison of cold water, hot water and air conditioning in nursing homes, retirement homes and hospitals. The results obtained show that the method CSR April 4, 2000 restricts the search to L. pneumophila permitting, than the method EN ISO II73I-I: 2008, to carry out a risk assessment well targeted to the actual pathogen. The culture method for the detection of $L$. pneumophila allows you to not only prevention, but also to implement a series of targeted interventions following the directions of the legislation.

\section{INTRODUZIONE}

Le legionelle sono bacilli Gram-negativi, di dimensioni variabili, scarsamente colorati, aerobi, privi di capsula, asporigeni, provvisti di uno o due flagelli, ossidasi e catalasi positivi, ureasi negativi. Necessitano per il loro sviluppo della presenza di cisteina e di un'elevata concentrazione di ferro ed utilizzano gli amminoacidi, ma non gli zuccheri, come fonte di carbonio e di energia.

Studi tassonomici hanno dimostrato che la famiglia delle Legionellaceae comprende un solo genere: la Legionella con 48 specie e più di 70 sierogruppi. Circa metà di queste specie e dei sierogruppi sono stati implicati in malattie dell'uomo, gli altri sono stati trovati in fonti ambientali.

Il termine "legionellosi" comprende tutte le forme morbose causate da microrganismi del genere Legionella.
La Legionella pneumophila è l'agente eziologico di quasi 1' $80 \%$ di tutte le infezioni polmonari; i sierotipi 1 e 6 sono quelli isolati più comunemente (2). La legionellosi è stata descritta la prima volta nel luglio del 1976, in seguito ad un'epidemia insorta tra i partecipanti ad un raduno di reduci della guerra del Vietnam svoltosi in un hotel di Philadelphia (Convention of American Legion). In quell'occasione, 221 persone contrassero questa forma di polmonite precedentemente non conosciuta e 34 morirono. La fonte di contaminazione batterica fu identificata nel sistema di aria condizionata dell'albergo (3).

L'acqua costituisce il principale serbatoio ambientale della Legionella. L'inquinamento termico delle acque rappresenta uno dei principali fattori favorenti la crescita; altri fattori importanti sono l'acidità, la presenza di fonti di nutrimento e la

\section{Corresponding author: Salvo Torrisi}

Via Defendente Sacchi, 25 - 27100 Pavia

Tel.: 333 4|4I672 - Fax: 013। 88I633

E-mail: salvohornet@alice.it 
presenza di altre forme di microrganismi. Inoltre, sedimenti organici, ruggini, depositi di materiali sulle superfici dei sistemi di stoccaggio e distribuzione delle acque facilitano l'insediamento della Legionella.

In teoria, il sistema definitivo di prevenzione della legionellosi consisterebbe nella completa eradicazione del batterio dall'ambiente. Questo approccio di fatto è irrealizzabile a causa della larghissima diffusione della Legionella nelle acque.

La forma di controllo più efficace consiste nel prevenire il problema intervenendo nella catena di trasmissione della malattia sul maggior numero di punti possibili.

Per questo, la legionellosi si pone come un serio problema di salute pubblica, perché costituisce un elemento di rischio in tutte le situazioni in cui le persone sono riunite in uno stesso ambiente, come avviene in case di cura, residenze per anziani, ospedali, piscine, stabilimenti termali e altri ambienti comunitari, nei quali è in funzione un sistema di condizionamento, di umidificazione o di trattamento dell'aria o di ricircolarizzazione delle acque.

Non è documentata la trasmissione uomo-uomo. La Legionellosi viene normalmente acquisita per via respiratoria mediante inalazione di aerosol contenente legionelle, oppure di particelle derivate per essiccamento. Le goccioline si possono formare sia spruzzando l'acqua che facendo gorgogliare aria in essa, o per impatto su superfici solide. Nell'uomo, le modalità di accumulo nel sistema respiratorio di goccioline di aerosol dipendono in primo luogo dalle loro dimensioni: più piccole sono le dimensioni delle gocce più queste sono pericolose.

Goccioline di diametro pari o superiore a $10 \mu \mathrm{m}$ si arrestano nel naso e nella gola, quelle di diametro compreso tra i 5 e i 10 $\mu \mathrm{m}$ si accumulano nelle vie respiratorie, sia superiori che inferiori.

Goccioline di diametro compreso tra i 2 e i $5 \mu \mathrm{m}$ si depositano nei polmoni, prevalentemente nelle ramificazioni bronchiali, infine al di sotto dei $2 \mu \mathrm{m}$ le goccioline raggiungono le superfici di scambio sangue-aria degli alveoli.

L'infezione da Legionella può essere asintomatica o dare luogo a due distinti casi clinici: Febbre di Pontiac che si manifesta in forma simil-influenzale dopo un incubazione di $24-48$ ore, senza interessamento polmonare e con risoluzione spontanea in 2-5 giorni; Malattia dei Legionari, patologia multisistemica con polmonite rapidamente progressiva.

\section{SCOPO DEL LAVORO}

Lo scopo principale del presente lavoro è quello di valutare la presenza di Legionella spp e di Legionella pneumophila secondo i diversi approcci proposti dalle Linee Guida delle Regioni Lombardia e Piemonte in termini di valutazione e prevenzione del rischio legionellosi in ambito nosocomiale.

In particolare le "Linee Guida Prevenzione e Controllo della Legionellosi in Lombardia" (Decreto n. 1751 del 24 febbraio 2009) si prefiggono come obiettivo quello di valutare la presenza dell'agente patogeno Legionella pneumophila, così da poter attuare in modo preventivo le misure di controllo adeguate. Le suddette Linee Guida hanno come riferimento differenti documenti tematici o generali emanati a livello nazionale quali:

- "Linee guida per la prevenzione e il controllo della legionellosi" della Conferenza StatoRegioni del 4 aprile 2000;

- "Linee guida recanti indicazioni ai laboratori con attività di diagnosi microbiologica e controllo ambientale della legionellosi";

- "Linee guida recanti indicazioni sulla legionellosi per i gestori di strutture turistico-ricettive e termali" della Conferenza Stato-Regioni del 13 gennaio 2005;

- "Linee guida per la definizione di protocolli tecnici di manutenzione predittiva sugli impianti di climatizzazione" della Conferenza StatoRegioni del 5 ottobre 2006;

- Disposizioni del Decreto Legislativo 81/2008 in materia di tutela della salute e sicurezza nei luoghi di lavoro.

Tabella I. Confronto fra le malattie causate da Legionella.

\begin{tabular}{|c|c|c|}
\hline & Malattia dei Legionari & Febbre di Pontiac \\
\hline \multicolumn{3}{|l|}{ Epidemiologia } \\
\hline Presentazione & Epidemica, sporadica & Epidemica \\
\hline Frequenza degli attacchi & $<5$ & $>90$ \\
\hline $\begin{array}{l}\text { Diffusione per contatto uomo- } \\
\text { uomo }\end{array}$ & No & No \\
\hline Malattia polmonare cronica & $\mathrm{Si}$ & No \\
\hline Periodo di insorgenza & $\begin{array}{l}\text { Malattia epidemica nella tarda } \\
\text { estate o in autunno; malattia } \\
\text { endemica durante l'anno. }\end{array}$ & Durante tutto l'anno \\
\hline \multicolumn{3}{|l|}{ Manifestazioni cliniche } \\
\hline Periodo di incubazione (giorni) & $2-10$ & $1-2$ \\
\hline Polmonite & $\mathrm{Si}$ & No \\
\hline Decorso & Terapia antibiotica necessaria & Autolimitantesi \\
\hline Mortalità (\%) & $\begin{array}{l}15-20 \text {; maggiore se la diagnosi } \\
\text { c̀ ritardata }\end{array}$ & $<1$ \\
\hline
\end{tabular}


Le Linee Guida "Raccomandazioni per la sorveglianza, la prevenzione e il controllo delle polmoniti da Legionella nelle strutture sanitarie piemontesi pubbliche e private" (Documento n.109 del 4 marzo 2008) si prefiggono come obiettivo quello di valutare la presenza di Legionella spp, così da poter proporre un set minimo omogeneo di procedure per la prevenzione e il controllo della legionellosi.

Le suddette Linee Guida definiscono la prevenzione del rischio legionellosi sulla base dei seguenti punti:

a) programmi di prevenzione ad hoc per singole strutture sulla base della carta di rischio;

b) sistemi di sorveglianza clinica da parte del medico di struttura;

c) interventi immediati in occasione della comparsa di casi o cluster epidemici;

d) valutazione periodica della contaminazione ambientale al fine della sorveglianza sanitaria;

e) verifica dell'efficacia dei programmi di bonifica attuati nel breve, medio e lungo periodo attraverso la sorveglianza sui casi e la ripetizione di indagini epidemiologiche correlate.

\section{METODI ANALITICI}

I metodi analitici utilizzati sono stati i seguenti:

- metodo UNI EN ISO 11731-2:2008. Qualità del-

l'acqua: ricerca e conta di Legionella

- metodo per filtrazione diretta su membrana per acque a basso contenuto batterico;

- ricerca e determinazione di Legionella pneumophila in acque destinate al consumo umano (procedura di prova secondo CSR 4 aprile 2000).

Per analisi microbiologica delle acque si intende l'individuazione dei microrganismi presenti in esse, generalmente congiunta alla loro valutazione quantitativa.

Ciò si ottiene mediante tecniche analitiche basate sulla riproduzione dei microrganismi considerati espressione del grado di inquinamento cui le acque sono soggette, su terreni colturali idonei alla loro crescita.

Nel caso particolare delle acque superficiali attraverso l'analisi microbiologica se ne definisce la qualità.

L'esame microbiologico delle acque in generale e delle acque superficiali in particolare, si basa essenzialmente sulla possibilità di coltivare su idonei terreni e in idonee condizioni colturali i batteri contenuti nell'acqua in esame, utilizzando particolari metodologie finalizzate alla individuazione di specie o di gruppi microbici che si ritengono significativi per la formulazione di un giudizio igienico-sanitario o di qualità delle acque in esame.
La metodica utilizzata per il monitoraggio dei punti di prelievo è stata la filtrazione per membrana (MF). Il metodo MF è comodo e utile per la sua praticità $(1)$.

Tale metodica prevede il campionamento di 1000 $\mathrm{ml}$ di acqua: di questi sono prelevate 2 aliquote da $10 \mathrm{ml}$ ciascuna mentre la restante parte è filtrata su due membrane di nylon del diametro di $47 \mathrm{~mm}$ (dimensione dei pori $0.2 \mu \mathrm{m}$ ) per concentrare la microflora presente.

La microflora trattenuta dal filtro è eluita nei 10 $\mathrm{ml}$ di acqua trattenuti all'inizio e concentrata con centrifugazione.

Per minimizzare la crescita di organismi diversi da Legionella, una quota della sospensione risultante è sottoposta a trattamento con acido (HCL$\mathrm{KCL}$ ) o a trattamento termico; questa fase è la più critica di tutto il processo e può influire in modo significativo sulla percentuale di recupero delle cellule batteriche.

Le quote trattate e non, sono seminate su piastre di terreno selettivo MWY (Wadowsky Yee Medium) o GVPC agar (Glicina, Vancomicina, Polimixina B, Cicloeximide) per le specie Legionella e poste in incubatore.

Al termine dell'incubazione, le colonie che assumono aspetti caratteristici sui terreni selettivi sono considerate sospette per specie Legionella e contate.

Le colonie sospette per Legionella sono poste in subcoltura sia su CYE agar base (Charcoal Yeast Extract) sia su BCYE agar (Buffered Charcoal Yeast Extract) con supplemento di 1-cisteina e ferro per verificare la crescita differenziale, e il numero dei microrganismi confermati è calcolato per 1 litro di campione.

Qualora sia necessario procedere con le fasi di identificazione fino all'individuazione del siero gruppo sono disponibili test biochimici e sierologici.

\section{RISULTATI}

Avendo come riferimento le Linee Guida nazionali (ricerca e determinazione di Legionella pneumophila in acque destinate al consumo umano procedura di prova secondo la Conferenza StatoRegioni del 4 aprile 2000) ed internazionali (metodo UNI EN ISO 11731-2:2008 - Qualità dell'acqua: ricerca e conta di Legionella - metodo per filtrazione diretta su membrana per acque a basso contenuto batterico), sono stati effettuati controlli comparativi sugli impianti di climatizzazione, di riscaldamento ed erogazione dell'acqua in alcune strutture di ricovero per anziani, ospedali, case di cura e residenze sanitarie assistite in Piemonte e Lombardia.

I controlli, eseguiti in periodi stagionali diversi (inizio autunno 2009/2010 e tarda primavera 
2010), hanno permesso di trarre indicazioni il più possibile verosimili sullo stato effettivo delle strutture e potere così programmare i controlli successivi nel momento migliore dal punto di vista preventivo.

Inoltre, sono stati effettuati controlli in situazioni funzionali diverse degli impianti in modo da poter verificare un'eventuale proliferazione microbica in situazioni diverse da quelle di normale utilizzo. Viene presentata una serie di dati analitici, a contributo della definizione dello stato dell'arte in tema di legionellosi per una sempre più capillare prevenzione, soprattutto in quei luoghi di ricovero e/o cura ove sono presenti soggetti particolarmente a rischio come bambini ed anziani.

In tutti i casi sono stati effettuati prelievi:

1. dall'impianto di produzione dell'acqua calda e anche dell'acqua fredda in tarda primavera;

2. dall'impianto di climatizzazione.

I punti di prelievo sono stati definiti in base a quanto riportato nelle Linee Guida.

E stato eseguito un prelievo di controllo nei punti interni all'impianto di erogazione dell'acqua potabile (rami morti, depositi calcarei, tubazioni, incrostazioni).

I dati analitici raccolti sono rappresentati nelle seguenti figure.

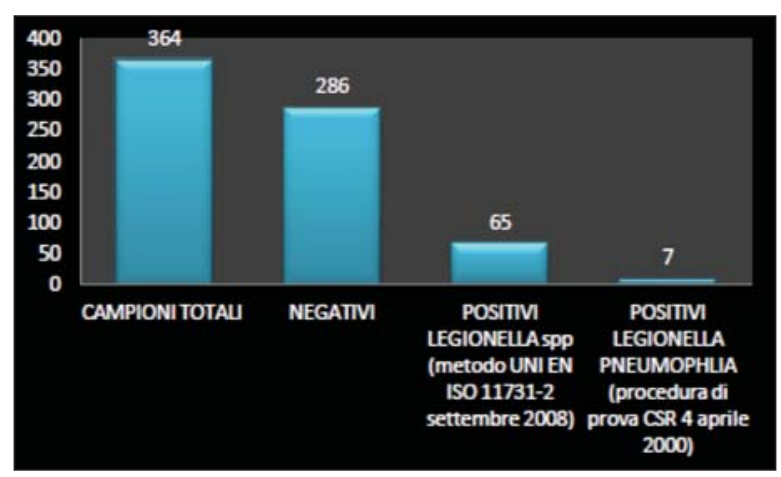

Figura I. Risultati ottenuti dal campionamento d'impianti di erogazione dell'acqua fredda.

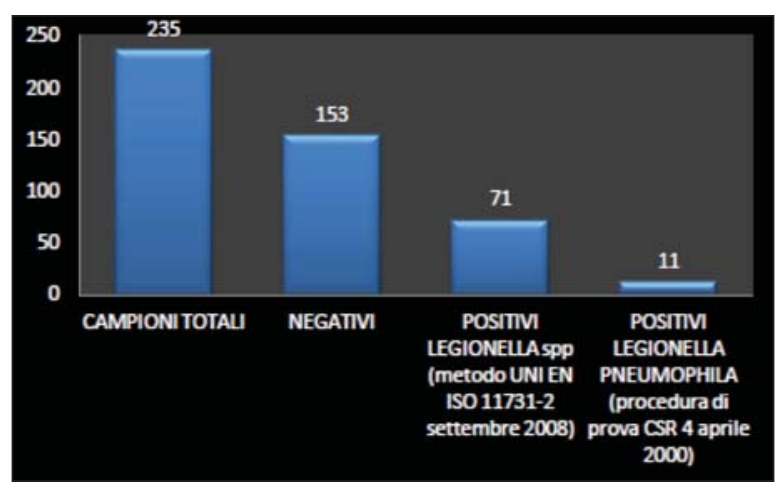

Figura II. Risultati ottenuti dal campionamento d'impianti di erogazione dell'acqua calda.

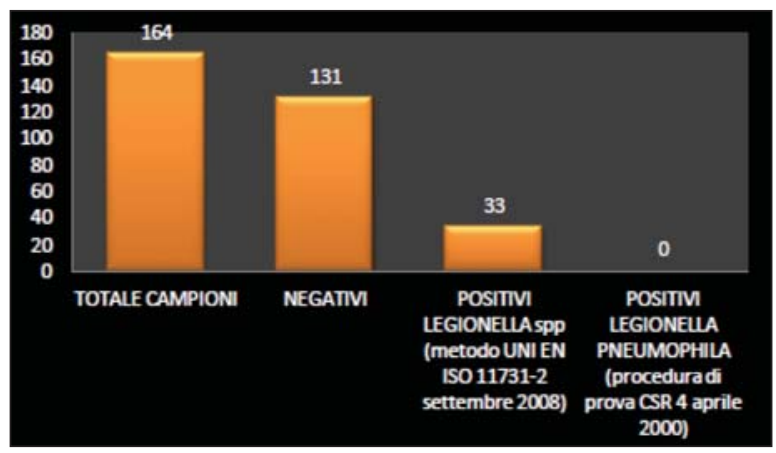

Figura III. Risultati ottenuti dal campionamento dei sistemi di climatizzazione.

Tabella 2. Concentrazione (UFC/L) di Legionella pneumophila in situazioni funzionali diverse da quelle di normale utilizzo.

\begin{tabular}{lc}
\hline Stato dell'Impianto & Concentrazione (UFC/L) \\
\hline Non funzionante o fermo & $5 \times 10^{3}$ \\
\hline Funzionante a regime & $10^{2}-10^{3}$ \\
\hline Inizio rete & 0 \\
\hline
\end{tabular}

\section{CONCLUSIONI}

I risultati ottenuti mediante analisi effettuata sui campioni di acqua hanno permesso di fare le seguenti considerazioni:

1) la Conferenza Stato-Regioni si è dimostrata una valida guida da seguire per la ricerca di Legionella pneumophila, raccomandando di fare prevenzione soprattutto in tema di legionellosi, poiché si tratta di una forma d'infezione "emergente" per il nostro paese;

2) la ricerca di Legionella, effettuata mediante il metodo colturale, con modi e tempi seri e coerenti, permette di ottenere risultati significativi. In tal modo, dai risultati ottenuti si ha quindi la possibilità di fare non solo prevenzione, ma anche di attuare tutta una serie d'interventi cautelativi di bonifica seguendo le indicazioni della normativa; inoltre è anche possibile coordinare il dato clinico con quello analitico, ed infine elaborare tutti i dati con il supporto degli strumenti statistici forniti dalla normativa.

Altro aspetto rilevante che emerge da questo studio è lo stato manutentivo dell'impianto, inteso non tanto come vecchiezza quanto come effettiva manutenzione programmata ed eseguita nel corso degli anni.

Infatti, dalle analisi effettuate risulta una ricerca e conferma di Legionella negli impianti di erogazione dell'acqua calda di strutture di recentissima costruzione ma senza un serio ed eseguito programma di manutenzione periodica.

Altro aspetto da considerare è il cambiamento di stato funzionale degli impianti, che può causare problemi. 
La Tabella 2 dimostra in modo inequivocabile come qualsiasi forma d'intervento sullo stato dell'impianto possa alterare anche in modo irreparabile equilibri raggiunti nel corso di svariati anni e instauratisi anche prima che si prendesse in considerazione il problema Legionella.

Tali equilibri comprendono certamente le caratteristiche chimico-fisiche dell'acqua immessa, la collocazione geografica della struttura, i materiali utilizzati per tubature e impianto, le caratteristiche di estensione dell'impianto stesso, l'età e i conseguenti depositi presenti lungo il percorso.

Infine, appare importante evidenziare come la procedura di prova secondo la Conferenza StatoRegioni del 4 aprile 2000 circoscriva il campo di ricerca a Legionella pneumophila permettendo, rispetto al metodo UNI EN ISO 11731-1:2008, di effettuare una prevenzione mirata all'effettivo agente patogeno.

\section{BIBLIOGRAFIA}

1. Health Protection Agency. National Standard method: General technique for the detection of bacteria by membrane filtration W1. London: Health protection Agency; 2003.

2. Murray PR, Rosenthal KS, Pfaller MA. Microbiologia Medica (Quinta Edizione - 2008). Edizione italiana sulla quinta edizione inglese 2008 -E.M.S.I. - Roma. Edizione italiana coordinata da: Giovanni Fadda, Cartesio Favalli, Giorgio Palù.

3. Puckorius PR. Update on legionnaires' Disease and cooling Systems: Case History Reviews - What Happened/What to do and current Guidelines, ASHRAE transaction. ASHRAE, Atlanta,USA. 1999; 105: 2. 